\title{
Chaos-Directed Genetic Algorithms for Water Distribution Network Design: An Enhanced Search Method
}

POOJITHA S. N ( $\sim$ pooji.pujitha@gmail.com )

Indian Institute of Technology Bombay https://orcid.org/0000-0003-2721-9224

Vinayakam Jothiprakash

Indian Institute of Technology Bombay

B. Sivakumar

Indian Institute of Technology Bombay

\section{Research Article}

Keywords: Water distribution network, Optimization, Genetic algorithm, Chaos-based evolutionary algorithms, Ergodicity, Non-sequential and sequential approaches

Posted Date: July 6th, 2021

DOI: https://doi.org/10.21203/rs.3.rs-574714/v1

License: (c) (1) This work is licensed under a Creative Commons Attribution 4.0 International License. Read Full License 


\title{
Chaos-Directed Genetic Algorithms for Water Distribution Network Design: An Enhanced Search Method
}

\author{
S. N. Poojitha ${ }^{1}$, V. Jothiprakash ${ }^{2}$, B. Sivakumar ${ }^{3}$ \\ ${ }^{1,2,3}$ Department of Civil Engineering, Indian Institute of Technology Bombay, Powai, \\ Mumbai, Maharashtra 400076, India. \\ E-mails: pooji.pujitha@gmail.com, vprakash@iitb.ac.in, b.sivakumar@iitb.ac.in
}

\begin{abstract}
The design of a water distribution network (WDN) is an ever-challenging problem. Development and application of optimization techniques for WDN design have been an important area of research. Recently, the introduction of chaos theory-based evolutionary algorithms (EAs) in addition to traditional random-based ones has elevated the scope for further improving the performance of EAs. The present study proposes a chaos-directed genetic algorithm (CDGA) by incorporating chaos ergodicity in GA mechanics for WDN optimal design. Two novel frameworks, the non-sequential approach (NSA) and sequential approach (SA) are introduced. The influence of chaotic systems with high dimensionality maps in improving the search efficacy of GA when compared to the low dimensionality maps is explored. Considering four widely studied WDN benchmark problems, the performance of the proposed GA and CDGA models is evaluated. From the results, it is observed that the CDGA models outperform GA with better search efficacy, requiring fewer function evaluations to locate the optimal solution. Also, concerning the different chaotic maps used in the present study to induce chaos ergodicity, the results highlight the usefulness of chaos-directed search in improving the computational efficiency of GA. From the computational results, the study suggests the usage of a chaotic system with other bio-inspired techniques for their improved search and computational efficiency.
\end{abstract}


Keywords: Water distribution network; Optimization; Genetic algorithm; Chaos-based evolutionary algorithms; Ergodicity; Non-sequential and sequential approaches

\section{Introduction}

Water distribution networks (WDNs) play a key role in socio-economic development. They are generally large complex systems made up of different hydraulic components that interact with each other in a nonlinear manner. The design of WDNs aimed at accomplishing hydraulic, functional, and economic aspects is often a highly challenging problem. The complexity in the design increases with an increase in the number of decision variables. It is a combinatorial optimization problem and is also a familiar nondeterministic polynomial-time hard problem (Reca and Martinez 2006).

During the past several decades, an enormous amount of research has been carried out for WDN design. Many efficient optimization techniques ranging from the scale of classical optimization techniques to heuristic and metaheuristic search techniques have been developed (e.g. Alperovits and Shamir 1977; Gessler 1985; Lansey and Mays 1989; Dandy et al. 1996; Ezzeldin et al., 2014; Zheng et al. 2014; Fallah et al. 2019). In recent decades, the application of metaheuristic optimization techniques such as genetic algorithm (GA), differential evolution (DE), particle swarm optimization (PSO), harmony search (HS), cuckoo search (CS), and crow search algorithm (CSA), for WDN optimal design have gained significant momentum due mainly to their versatility and ease in application. Among the many metaheuristic optimization techniques, GA is one of the most widely used techniques (e.g. Simpson et al. 1994; Gupta et al. 1999; Vairavamoorthy and Ali 2000; Zyl et al. 2004; Kadu et al. 2008; Haghighi et al. 2011; Mora-Melia et al. 2013; Johns et al. 2014).

Genetic Algorithm (GA) is a bio-inspired metaheuristic, evolutionary algorithm (EA).

The evolutionary process of GA in the traditional sense is governed by the initial population that is generated randomly. It is a random, yet structured algorithm, where an exchange of 
information takes place among the population members (that are chosen through a selection mechanism) over the generations through its crossover and mutation operators that work on the probabilistic rules and a random phenomenon (Goldberg and Kuo 1989). Although the algorithm generally works well, it also has certain drawbacks. For instance, (i) as the search procedure is a mere dependence of random walks in a random direction, the occurrence of an optimal solution is skeptical at every trial considered (Savic and Walters 1997; Keedwell and Khu 2005), and (ii) with an increase in the number of decision variables, GA becomes computationally expensive with a high chance of stagnating at local optima due to an insufficient exploration of the search space (Ingu and Takagi 1999; Reca and Martinez 2006; Cheng et al. 2008; Zheng et al. 2011; Ali et al. 2018). Owing to these drawbacks, many attempts have been made in recent decades to improve the searchability and computational efficiency of GA.

Murphy and Simpson (1992) were the first to apply GA for the optimal design of WDNs which was followed by Simpson et al. (1994). In these studies, binary encoding was used to represent the decision variables. Later, Dandy et al. (1996) and Savic and Walters (1997) improvised the binary GA using gray coding. The problems associated with binary alphabet decoding are alleviated by Gupta et al. (1999), followed by Vairavamoorthy and Ali (2000) by formulating a real-coded GA. The other GA models developed include GA with an enhanced operator's (Montesinos et al. 1999; Reca and Martinez 2006; Johns et al. 2014), messy GA with building block filtering process (Wu and Simpson 2001), hybrid GA models (Zyl et al. 2004; Cisty 2010; Haghighi et al. 2011), GA fed with a healthier initial population (Vairavamoorthy and Ali 2005; Keedwell and Khu 2005), modified-GA with a critical path scheme (Kadu et al. 2008), pseudo GA with integer coding scheme (Mora-Melia et al. 2013), GA with a novel decision variables representation (Cimorelli et al. 2020), and many more. 
While various such approaches have and continue to be formulated, attempts to

combine the concepts of chaos theory (see Lorenz (1963) and May (1976) for early studies on the theory) with the metaheuristic optimization techniques emerged in the meantime in diverse fields of engineering. Such attempts elevated the scope for improving the performance of EAs; see, for example, Mozaffari et al. (2018) for a detailed investigation on the effects of using different chaotic maps on the diversification or intensification features of EAs. In the field of water resources engineering, some of the studies on chaos-based GA models include, Yuan et al. (2002), Cheng et al. (2008), Arunkumar and Jothiprakash (2013).

Despite the chaos theory-based attempts and outcomes to enhance the effectiveness and efficiency of EAs, the use of a chaotic approach has not been, to the best of our knowledge, explored for the optimal design of WDNs. It is important to note, however, that chaotic maps, within a certain range depending on the system characteristics, evolve through every possible state (Ammaruekarat and Meesad 2011). The dynamic evolution through all the possible states is particularly useful in optimization since it keeps the chance of reaching the global point of interest always high. This advantage of chaotic maps provides a great opportunity to completely leverage it in WDN optimization studies, through its introduction in GA that uses the inherent information for the evolution over generations. In this sense, the present study offers a novel approach to replace the entire random phenomenon of GA with chaos ergodicity, to achieve enhanced searchability and computational efficiency.

In most of the chaos theory-based water resources-related optimization models discussed above, the Logistic series was used. Further, in the studies where chaos maps were used in more than two operators, Logistic sequences with different initial conditions were used. As chaotic maps are highly sensitive to initial conditions, the chaos ergodicity may not be employed for the entire qualitative search when the operators are driven with different chaotic dynamics (i.e., with different initial conditions). In this regard, to retain the chaos ergodicity 
throughout the optimization process and to analyze its effects on the convergence properties of GA, the present study uses the chaotic map with the same initial characteristics throughout the optimization process. Essentially, the present study differs from the previous ones in the way the chaos ergodicity is simulated in GA random search mechanisms. Thus, the Chaos-directed Genetic Algorithm (CDGA) models are formulated introducing two novel frameworks for chaotic sequence (i.e., time series) allocation. They are (i) the non-sequential approach (NSA), where the chaotic sequence is pre-allocated at every random phase of GA, for the entire generation size $G_{s}$; and (ii) the sequential approach (SA), where, over generations, the chaotic sequence is assigned successively at every random phase of GA.

It is important to note that a chaotic system with high dimensionality may allow a more diversified search when compared to a chaotic system with low dimensionality, especially in terms of the exploration in the search space (i.e. greater search effectiveness). Whether this is indeed true has not been examined thus far, since most of the studies that have attempted a chaotic search for the design of water resources-related optimization problems have essentially used the Logistic time series, which has very low dimensionality (correlation dimension is about 0.50) (Grassberger and Procaccia 1983). To address this issue, the present study explores the use of slightly more complex chaotic systems with higher dimensionality. To this end, two other chaotic systems, namely the Henon map (Henon 1976) (with a dimension of 1.22) and the Lorenz map (Lorenz 1963) (with a dimension of 2.06), are considered. The effectiveness of the CDGA models is demonstrated by first applying them to three well-studied benchmark problems: the Two-loop network (TLN) (Alperovits and Shamir 1977), the Bakryan network (BRN) (Lee and Lee 2001), and the Goyang network (GYN) (Kim et al. 1994). Then with the best of all the models, scalability is checked using the large problem, the Balerma irrigation network (BIN) (Reca and Martinez 2006). 

the CDGA models formulated are discussed in Section 2. This section also compiles the combined optimization and simulation framework, and the details of the benchmarks problems considered in the study. The sensitivity analysis and computational results are presented in

Section 3. Section 4 highlights and discusses the key points observed from the computational analysis and results. The validation of the computationally efficient best model's scalability using a large network, BIN is also presented in Section 4. Finally, in Section 5 the major conclusions drawn from the study are highlighted.

\section{Methods and Methodology}

\subsection{Genetic Algorithm (GA) Model with Random Search Phenomenon}

Genetic Algorithm simulates the Darwinian principle of genetic inheritance (Goldberg and Kuo 1989). In GA, the evolution of the population takes place over generations by refining them through selection (reproduction), crossover, and mutation operators. There exist different mechanisms for GA operators. The present study considers the real-coded GA in its traditional form with truncation selection mechanism, single-point crossover, and bitwise mutation operators. The main motive here is to simulate the chaos ergodicity in the traditional form of GA and to compare its performance with the other GA and metaheuristic optimization techniques reported in the literature, for handling the WDN optimization problem.

In the present case of WDN design, the main objective is to minimize the design cost subjected to the constraint of the minimum head criterion. There are different constraint handling methods with each having its advantages and disadvantages. In evolutionary computation and other optimization frameworks, penalty functions that penalize the infeasible solutions are widely considered. In the present study, the static penalty function that is easy and simple to implement (Michalewicz and Schoenauer 1996) is considered. Thus, the fitness function, $F_{f}$, is defined as the sum of the pipe cost, $C$, and the static penalty function, as follows: 
where $d$ and $l$ are the diameter and length of pipes respectively, $N_{D}$ is the number of decision variables (number of pipes whose diameters should be optimally designed), $N_{n}$ is the number of nodes, $P M$ is the penalty multiplier (chosen through sensitivity analysis; discussed below), and $H_{\min }$ and $H_{i}$ are the minimum head requirement and the head available at the $i^{\text {th }}$ node.

\subsubsection{GA Mechanism}

The optimization procedure of GA begins with the generation of a random set of the initial population. The genes or decision variables, $D$, in every chromosome of the population maps to a diameter within in the commercially available diameter set $(C D S)$ as follows:

$D_{i j}=L_{i j}+\left(U_{i j}-L_{i j}\right) \times r_{i j} \quad i=1,2, \ldots, P_{s} ; j=1,2, \ldots, N_{D}$

where $D_{i j}$ is the $j^{\text {th }}$ decision variable of the $i^{\text {th }}$ chromosome, $U$ and $L$ are the upper and lower bounds (which equals to the maximum and minimum count of the number of commercially available pipe diameter options, $\left.N_{C D}\right), r$ is the random number, and $P_{s}$ is the population size.

Once the initial set of the random population is generated, $2 \%$ of the elite or best chromosomes (evaluated based on the fitness function) are saved for the next generations without undergoing any GA operations. From the population set that is left out, the best chromosomes are reproduced or selected using the truncation operator. Under this operation, based on the predefined truncation probability, the best chromosomes are retained and the remaining ones are discarded. There is no random phenomenon involved in this operator. The truncation probability depends on the crossover probability, $P_{c}$, which is calibrated in the present study through sensitivity analysis.

Following the reproduction operator, the best-retained chromosomes undergo crossover to generate offsprings of size equal to the discarded ones, conserving the fixed population size, $P_{s .}$ At each time of crossover operation, two new chromosomes (offsprings) are generated using 
172 two parent chromosomes, $P C 1$, and $P C 2$, which are chosen randomly from the 173 retained chromosomes, $P_{r}$, as follows:

$174 \quad P C 1=r_{1} \times P_{r} ; \quad P C 2=r_{2} \times P_{r}$

175 where $r_{1}$ and $r_{2}$ are the numbers generated randomly. After $P C 1$ and $P C 2$ are chosen, they are rounded to the nearest whole number. For every run of the crossover operation, the crossover 177 point $C P$ is chosen randomly between 1 and $\left(N_{D}-1\right)$, as follows:

$C P_{k}=r_{k} \times\left(N_{D}-1\right)$

$$
k=1,2, \ldots, P_{r} / 2
$$

Thus, in the crossover operation, the random phenomenon is used in two steps: in the selection of the parent chromosomes and the selection of a crossover point.

Once the $P_{s}$ is retained by reproducing the offsprings, the mutation operator is performed to maintain the diversity in the population set. The mutation chromosome, $M C$, and the mutation variable, $M V$ (the particular decision variable (gene) of the selected $M C$ ), are chosen randomly as follows:

$M C_{l}=r_{l 1} \times P_{s} ; \quad M V_{l}=r_{l 2} \times N_{D} \quad l=1,2, \ldots, M$

The crossover and mutation probabilities, $P_{c}$ and $P_{m}$, define on average the number of chromosomes and genes that undergo crossover and mutation operations (Goldberg and Kuo 1987; Dandy et al. 1996). In the present study, for ease in application, the number of genes, $M$, that undergo mutation operation from the entire population set is estimated by considering the product of $P_{m}, P_{s}$, and $N_{D}$. Once $M V$ of an $M C$ is randomly chosen, it is swapped using the swapping variable, $S V$, which is also chosen randomly as follows:

193 Thus, under the mutation operation, the random phenomenon is used in three steps, i.e., in the selection of $M C, M V$, and $S V$. In total, the GA model formulated in the present study involves 
random phenomena in the generation of the initial population, the crossover, and mutation mechanisms. This makes the convergence to an optimal solution skeptical and uncertain at every trial of execution, and may even increase the computational burden with an increase in decision variables. Therefore, there is still a lot of scope for improving GA's performance.

\subsection{Chaos-directed Genetic Algorithm (CDGA) with chaotic search phenomenon}

In the present study, to enhance GA's search capability and computational efficiency, chaos ergodicity is simulated in GA's search mechanism using chaotic maps. The chaotic maps or systems are nonlinear deterministic systems that produce random-looking outputs. They are attractive with their clear patterns, and evolve within definite areas, with no repeating values in their time series. Chaotic systems are simple and sensitive to initial conditions, which is their essential characteristic. They are predictable in the short term due to their inherent determinism but not in the long run due to the nonlinear interdependency among the governing variables and sensitivity to initial conditions (Sivakumar 2017). Given these unique features of chaotic systems over random generators, in the present study, the chaos-directed genetic algorithm (CDGA) is formulated.

The CDGA model replaces every random mechanism in GA with the chaotic force, by assigning the chaotic sequence. In the present study, two novel frameworks are introduced to assign the chaotic sequence to GA operators, namely (i) non-sequential approach (NSA) and (ii) sequential approach (SA). The detailed mechanisms of these two approaches are shown in Fig. 1. In NSA, the assignment of the chaotic sequence is over each operator (involved with a random phenomenon) for the entire $G_{s}$, (i.e., non-sequential over generations). In SA, the assignment of the chaotic sequence is over generations, following every operator involved with a random phenomenon (i.e., sequential over generations). For both of these approaches, the initial population is generated using a chaotic sequence, $C S$, as follows:

$$
D_{i j}=L_{i j}+\left(U_{i j}-L_{i j}\right) \times C S_{k} \quad i=1,2, \ldots, P_{s} ; j=1,2, \ldots, N_{D} ; k=1,2, \ldots, P_{s} \times N_{D}
$$


where $K$ is the length of the chaotic sequence required for the initial population generation and is equal to $P_{s} \times N_{D}$. For the generation of offsprings, the parent chromosome, $P C$, that needs to undergo crossover operation is selected using a chaos-based equation:

$$
P C 1=C S_{l} * P_{r} ; \quad P C 2=C S_{l+1} * P_{r} \quad l=(K+1), \ldots, L_{n s} \text { or } L_{s}\left(G_{s}\right)
$$

where $L_{n s}=K+\left(P_{s}-P_{r}\right) \times G_{s}$ using NSA, and $L_{s}\left(G_{s}\right)=K+\left(P_{s}-P_{r}\right)+f\left(G_{s}\right)$ using SA which is the function of $G_{s}$. For $G_{s}=1, f(1)=0$, and for the last generation, $f\left(G_{s}\right)=D L_{s} \times\left(G_{s}\right.$ - 1), where $D L_{s}$ is the data length of the chaotic sequence allocated for a single generation using SA. The crossover point $C P$ is chosen using the chaotic sequence as follows:

$$
C P_{i}=C S_{m} \times\left(N_{D}-1\right) ; m=\left(L_{n s}+1\right) \text { or }\left(L_{s}\left(G_{s}\right)+1\right), \ldots, M_{n s} \text { or } M_{s}\left(G_{s}\right)
$$

where $M_{n s}=L_{n s}+\left(0.5\left(P_{s}-P_{r}\right)\right) \times G_{s}$ using NSA, and for SA, $M_{s}\left(G_{s}\right)=L_{s}\left(G_{s}\right)+$ $0.5\left(P_{s}-P_{r}\right)$. Following $C P$, for mutation operation, the mutation chromosome, $M C$ is chosen chaotically as:

$$
M C_{i}=C S_{i} \times P_{s} \quad i=\left(M_{n s}+1\right) \text { or }\left(M_{s}\left(G_{s}\right)+1\right), \ldots, N_{n s} \text { or } N_{s}\left(G_{s}\right)
$$

where for NSA, $N_{n s}=M_{n s}+\left(M \times G_{s}\right)$ and for SA, $N_{s}\left(G_{s}\right)=M_{s}\left(G_{s}\right)+M$. Following $M C$, the mutation variable, $M V$, is chosen as follows:

$M V_{j}=C S_{j} \times N_{D} \quad j=\left(N_{n s}+1\right)$ or $\left(N_{s}\left(G_{s}\right)+1\right), \ldots, Q_{n s}$ or $Q_{s}\left(G_{s}\right)$

$Q_{n s}=N_{n s}+\left(M \times G_{s}\right)$ for NSA, and $Q_{s}\left(G_{s}\right)=N_{s}\left(G_{s}\right)+M$, for SA. The swapping variable, $S V$, is chosen using:

$S V_{k}=C S_{k} \times N_{C D} \quad k=\left(Q_{n s}+1\right)$ or $\left(Q_{s}\left(G_{s}\right)+1\right), \ldots, R_{n s}$ or $R_{s}\left(G_{s}\right)$

Where for NSA, $R_{n s}=Q_{n s}+\left(M \times G_{s}\right)$, and for SA, $R_{s}\left(G_{s}\right)=Q_{s}\left(G_{s}\right)+M$. For a single generation, using $\mathrm{SA}, D L_{s}$ required is

$D L_{s}=L_{s}(1)+0.5\left(P_{s}-P_{r}\right)+3 M$ 

evolutionary search procedure with chaos ergodicity. In the present study, to emulate the chaos ergodicity, three chaotic maps are considered, namely, Logistic, Henon, and Lorenz maps whose characteristics in detail are enclosed in supplementary material, S_1. The corresponding CDGA models developed are CDGA-I, CDGA-II, and CDGA-III, respectively.

\subsection{Optimization and Simulation Model}

For the optimal design of WDNs, information on the following is generally considered

to be known a priori: layout of the network, pipe material, commercially available diameter set $C D S$, demand, and minimum pressure head requirement at the demand nodes. With this information, the main objective of the WDN optimal design is to choose meticulously an economical set of diameters for the pipes in the network, meeting completely the nodal demands at demand nodes. The fitness function $F_{f}$ is detailed in Equation (1). Here, for every possible solution set proposed by the optimization algorithm, the pressure head at every demand node is checked for meeting the minimum head criterion. Violation of the constraint if any, is penalized. The selection of the diameter for the pipes from the $C D S$ is ensured automatically, as presented in Equation (2). The other constraints such as balancing the energy and mass balance equations are ensured through a simulation model that simulates the actual hydraulic conditions of the WDN. In the present study, the EPANET software that works on the global gradient method, designed by Rossman (2000) is considered for simulation. Thus, the design algorithm for WDN is the combination of an optimization algorithm and a simulation model. The codes for both the GA and the CDGA models are written in MATLAB and are linked to the EPANET software using the MATLAB EPANET interface. tested on three benchmark networks TLN, BRN, and GYN. Also, the scalability of the best model is validated considering a 454-dimensional problem, BIN. 


\subsection{Benchmark Problems}

TLN is arguably the most widely used gravity-fed network with two loops. It is an 8dimensional problem, first introduced by Alperovits and Shamir (1977). The BRN is a 9dimensional rehabilitation network, first introduced by Lee and Lee (2001). The GYN is a 9 loop pumped medium-sized network with a single pump, and 30 pipes (Kim et al. 1994). Details of these three benchmark problems, in terms of the number of design parameters $N_{D}$, number of commercially available diameters $N_{C D}$, solution space available (i.e., number of possible combination of pipe sets from the commercially available data set), and the minimum residual pressures $H_{\min }$ to be maintained at the demand nodes, are presented in Table 1. Other layout and hydraulic details of these networks can be found in Geem (2006). The fourth network used is BIN, a large network with 454 pipes and 443 demand nodes. Each pipe of BIN has 10 commercially available diameter options resulting in a solution search space of $1.0 \times$ $10^{454}$. A pressure head of $20 \mathrm{~m}$ at demand nodes is desirable for BIN, to deliver the designed demands. The complete details of BIN are available in Reca and Matinez (2006).

\section{Analysis and Computational Results}

\subsection{Parameter Sensitivity Analysis}

The parameters of GA play a vital role in the convergence properties of the algorithm. An optimally chosen parameter set along with well-defined search guidance drives the algorithm towards global convergence. In the present study, to choose the optimal value for the parameters, a sensitivity analysis is performed. While performing the sensitivity analysis, the scale over which the parameters vary is chosen carefully. A too small or too large value of any parameter may not result in an efficient or effective search. For instance, for the population size, $P_{s}$, if a too low value is considered, the population gets saturated soon without proper exploration of the search space; if a too large value is considered, the population becomes redundant making the search highly random. To overcome these problems, in the present study, 
$P_{s}$ is varied in the moderate range of 50 to 500 for small to medium-sized problems, and 500 to 2500 for the large network. The probability of crossover, $P_{c}$, explicitly reflects the percentage of new chromosomes (offsprings) generated. A too-small $P_{c}$ means not much new information is generated to explore the new areas in the search space; a too-high $P_{c}$ loses useful information with the best fit chromosomes (an effective guiding medium). Thus, a moderate $P_{c}$, varying from 0.5 to 0.7 is considered. Once the $P_{c}$ is fixed, the truncation probability is considered as $\left(1-P_{c}\right)$. Although the leverage of inherent information with the randomized crossover operator payoff the search in unexplored areas, there is always a chance of overindulgence and missing important information (Goldberg and Kuo 1989). Therefore, a small percentage of the probability of mutation $P_{m}$ (as compared to $P_{c}$ ) in the range of 0.01 to 0.1 (Savic and Walters 1997; Kadu et al. 2008) is considered to induce diversity in the population. The too-high $P M$ makes the search stringent, which may lead to a loss of useful information involved with the infeasible solutions; on the contrary, a too low $P M$ value may concentrate the search around the infeasible regions. Accounting for these two complications, a moderate value of $P M$ that results through the sensitivity analysis is considered. Once all these parameters are fixed, the generation size, $G_{s}$, is chosen based on the convergence requirement of the algorithm.

It is important to note that the above parameters are problem-specific and modelspecific. Therefore, in the present study, the sensitivity analysis is carried out separately for each model with each of the benchmark problems considered.

\subsection{Results for Two-Loop Network (TLN)}

\subsubsection{GA Model}

At first, the sensitivity analysis for TLN is performed using the GA model. The optimal parameter set results from the analysis are presented in Table 1. With this optimal parameter set, the GA model is successful in converging to an optimal feasible design cost of $\$ 419,000$ by exploring only a small percentage of the solution space, i.e., $2.033 \times 10^{-4}$, with minimum 
function evaluations (MFEs) of 3,000, requiring an average computation time of $21.556 \mathrm{~s}$.

These results in comparison with the other models considered in the present study are presented in Table 1.

\subsubsection{CDGA-I Model}

Following the GA model, the CDGA-I model (i.e., with the Logistic map) is considered. Initially, to fix the fertility rate, $a$, of the Logistic equation, CDGA-I is run, by varying $a$ from 3.8 to 4.0 with a step size of 0.02 and with nine different initial values $X_{0}$, varying from 0.1 to 0.9. With the NSA and SA, it is found that $a=\{3.94,3.96,3.98,4.0\}$ are efficient in yielding an optimal cost of $\$ 419,000$. As the initial value $X_{0}$ of the Logistic equation can take any value in the range $(0,1)$, and the fact that the chaotic system is sensitive to even a fractional change in the initial value, $X_{0}$ is chosen randomly for the main analysis. Thus, the Logistic equation with $a=\{3.94,3.96,3.98,4.0\}$ and with a randomly chosen initial value is used to replace the random phases in GA in the two approaches, i.e., CDGA-I using NSA and CDGA-I using SA. Keeping $P_{s}, P_{c}$, and $P_{m}$ the same as that of the GA model, $G_{s}$ is varied from 100 to 200 ,

with a step size of 10 . At $a=3.94$, the Logistic sequence is found to be very efficient in obtaining the optimal cost for every combination of $G_{s}$ considered for both the approaches, as shown in Fig. 2(a). With NSA, at $G_{s}=\{150,200\}$, the MFEs required are 2,900, which is less than that of the random approach of the GA model $(3,000)$. At $a=3.96$, the optimal cost is obtained at every combination, except at $G_{s}=\{140,150\}$ of SA, as shown in Fig. 2(b). The MFEs required to locate the optimal cost are found to be less than the GA model at $G_{s}=120$, i.e., 2,900, using SA. At $a=3.98$, the CDGA-I model excels in attaining an optimal cost with a much lesser number of MFEs, i.e., 1,600, at $G_{s}=130$ using SA, as shown in Fig. 2(c). At $a=$ 4.0, the CDGA-I model converges to the suboptimum using NSA and SA at $G_{s}=120$ and $G_{s}=$ 100 , respectively. At $G_{s}=120$, using SA, compared to the GA model, a lesser number of MFEs are required i.e., 2,400 (see Fig. 2(d)). 
A summary of the best results of the sensitivity analysis using CDGA-I models is

presented in Table 1. Overall, the CDGA-I model is successful and efficient in exploring a small percentage of the solution space, i.e., $1.084 \times 10^{-4}$ to locate the optimal solution, with the MFEs of 1,600 , requiring a very less computation time of an average of $6.917 \mathrm{~s}$ as compared to that of the GA model.

\subsubsection{CDGA-II Model}

To check the impact of two-dimensional chaotic Henon map dynamics on the application of GA, the CDGA-II model is formulated. As the Henon map is two-dimensional $(x, y)$, the sequences of both dimensions are used. For a fixed $G_{s}$ of 400 , and with $P_{s}=\{53$, $102\}, P_{c}=\{0.5,0.7\}$, the CDGA-II model is found to be efficient in obtaining the optimal solution almost at every value of $P_{m}$ varying from 0.02 to 0.09 using both the NSA and SA. Fig. 2(e) to Fig. 2(1) shows the results with the $x$ and $y$ sequences, respectively. Using $x$ sequence, at $P_{s}=53$ with a combination of $P_{c}=0.5,0.7$ (see Fig. 2(e), (f)), the CDGA-II with NSA is successful in converging to the optimal solution with a lesser number of MFEs, 2,340 at $P_{m}=\{0.02,0.03\}$, and 1,820 , at $P_{m}=0.04$ respectively, which is less than that of the GA model $(3,000)$. When $P_{s}=102$ is considered, at both $P_{c}$ values $(0.5,0.7)$, for every combination of $P_{m}$, the optimal solution is located at the cost of an additional number of MFEs as compared to that of the GA model (see Fig. 2(g), (h)).

With the $y$ sequence, for almost all the combinations, the optimal solution of $\$ 419,000$ is obtained (as shown in Fig. 2(i), 2(j), 2(k), and 2(1)). With the combination of $P_{s}=53$, at $P_{c}$ $=0.5, P_{m}=0.02$ using SA (Fig. 2(i)), and at $P_{c}=0.7, P_{m}=0.03$ using NSA (Fig. 2(j)), a lesser number of MFEs 1,$924 ; 1,664$ is required for the convergence, when compared to the GA model $(3,000)$. With $P_{s}=102$, at $P_{c}=0.7, P_{m}=0.04$, using NSA, the optimal solution is located with fewer MFEs $(1,768)$ (see Fig. 2(1)). For every other combination of $P_{c}, P_{m}$, the MFEs required are more when compared to the GA model (see Fig. 2(k), (1)). The best results for the 
two approaches using the $x$ and $y$ sequences with the CDGA-II model are compiled in Table 2.

The main advantage of the CDGA-II model is, once the optimal parameter set of the algorithm is fixed, it excels in obtaining the optimal solution at every trial run with a $100 \%$ success rate. The best results of the CDGA-II model in comparison with the other models are presented in Table 1. Thus, overall, the CDGA-II model with non-sequential $y$ sequence outperforms the GA model in terms of a high success rate and with better computational efficiency in exploring a small percentage of the solution space, i.e., $1.128 \times 10^{-4}$ with an MFEs of 1,660 and with less average computation time of $8.007 \mathrm{~s}$ (see Table 1).

\subsubsection{CDGA-III Model}

Following the CDGA-II, the CDGA-III model with the three-dimensional Lorenz map is considered. All three sequences of the Lorenz map in combination with the NSA and SA are considered for the analysis. From the sensitivity analysis, for the NSA of the CDGA-III model using the $S$ sequence, the optimal parameter set is found to be $P_{s}=102, G_{s}=400, P_{m}=0.08$, and $P_{c}=0.5$. With this optimal parameter set, the algorithm converges to an optimal feasible cost of $\$ 419,000$ with the MFEs as high as 32,700 exploring the $2.216 \times 10^{-3}$ percentage of solution space with a minimum average computation time of 19.871 s. For the remaining sequences of the Lorenz map, with both approaches, the CDGA-III model converges to the local optimum. Although the CDGA-III model converges to the optimal solution with more function evaluations when compared to that of the GA model, the average time of computation is less. Further, similar to that of the CDGA-II model, if the optimal parameter set is fixed, the success rate of the CDGA-III model is $100 \%$, unlike the GA model.

Thus, from the results of sensitivity analysis for TLN, it is evident that the CDGA models are efficient in decreasing the computational effort. Especially, the CDGA models with the Logistic map and the Henon map (i.e., CDGA-I and CDGA-II) are efficient in decreasing 
the optimum feasible cost of $\$ 419,000$, over the GA model with the random search phenomenon.

\subsection{Results for Bakryan Network (BRN)}

\subsubsection{GA Model}

The optimal parameter set ascertained from the sensitivity analysis for BRN using the GA model is presented in Table 1. From the results, it is observed that the GA model is successful in converging to a design cost of $\$ 903,620$ (similar to the earlier studies by Lee and Lee (2001), Geem (2006)). For this optimal convergence, the GA model explores $9.330 \times 10^{-5}$ percentage of the solution space requiring 2,200 MFEs, with an average computation time of $74.988 \mathrm{~s}$, as presented in Table 1.

\subsubsection{CDGA Models}

The computational results of BRN using CDGA models for both the NSA and SA with varied $P_{m}$ values are presented in supplementary material S_2. The best results of the CDGAI and CDGA-II models along with their respective optimal parameter sets are presented in Table 1. It is observed that, unlike the GA model, the CDGA-I requires comparatively small $P_{s}$ and $G_{s}$ for convergence. And for locating the optimal cost of $\$ 903,620$, the CDGA-I model required very fewer MFEs (728), exploring a minimal percentage of the solution space (3.087 $\left.\times 10^{-5}\right)$, with an average computation time of $8.003 \mathrm{~s}$.

The CDGA-II model with both the sequences and with both the approaches succeeds in achieving an optimal solution with less computational effort as compared to the GA model. From Table 2, it is seen that the SA outperforms the NSA. Particularly, the sequential $x$ approach outperforms with much less computational effort exploring a very less percentage of the solution space, i.e., $4.411 \times 10^{-5}$, requiring fewer MFEs $(1,040)$ with an average computation time of 8.035 s (see Table 1). Using the CDGA-III model, only the $S$ sequence 
with the SA is successful, in converging to the optimal cost requiring MFEs of 6,600, exploring $2.799 \times 10^{-5}$ percent of the solution space, with an average computation time of $20.690 \mathrm{~s}$. computational effort as compared to the GA model, especially the CDGA-I, and CDGA-II.

\subsection{Results for Goyang Network (GYN)}

The optimal parameter set for GYN, obtained from the sensitivity analysis using the

GA, CDGA-I, and CDGA-II models is presented in Table 1. An improved feasible design cost $\times 10^{-22}$ percentage of the solution space with MFEs of 10,088 in an average computation time of $27.352 \mathrm{~s}$ to reach this optimal solution. The detailed computational results of the CDGA models with the NSA and SA are presented in supplementary material S_3. From the results, it is observed that the CDGA-I model outperforms the GA model in decreasing the search space, and thus the computational effort. At $a=3.98$ using the NSA, the CDGA-I model requires far fewer MFEs $(7,280)$ with an average computation time of $27.002 \mathrm{~s}$. The percentage of the solution space explored is only $5.881 \times 10^{-22}$ (see Table 1$)$.

The best computational results using the CDGA-II model with the $x$ and $y$ sequences are presented in Table 2. From the results, it is clear that the CDGA-II model requires relatively more MFEs when compared to the GA and CDGA-I models. However, with the best parameter set $\left(P_{s}=53, G_{s}=500, P_{m}=0.035\right.$, and $\left.P_{c}=0.7\right)$, using the non-sequential $y$ approach, on average, the time needed for executing the algorithm is less $(25.985 \mathrm{~s})$ when compared to that of the GA (27.352 s) and CDGA-I models (27.002 s). With this optimal parameter set, the percentage of the solution space explored by the CDGA-II model for converging to the optimal solution is $1.798 \times 10^{-21}$ (see Table 1 ). With the CDGA-III model, from the sensitivity analysis results, it is found that with the three sequences of the Lorenz map, both the approaches converge to a sub-optimal solution of $177,010,359 \mathrm{KRW}$. 

decreasing the computational effort as compared to the GA model.

\section{Discussions}

\subsection{Computational Analysis of CDGA Models}

An interesting feature observed from the computational analysis using the CDGA-I model with the Logistic map is that for any value of $a$, at $X_{0}=\{0.4,0.6\}$, convergence remains the same, including the number of function evaluations. Further, at $a=4.0$, the initial values $X_{0}=\{0.1,0.9\}$ have the same convergence properties. For all the three benchmark problems considered in this study, the CDGA-I model with $a=3.94,3.98$, and 4.0 is found to be efficient in converging to an optimal solution with a lesser number of function evaluations. Among $a=$ 3.94, 3.98, and 4.0, the Logistic sequence with $a=3.98$ is efficient in terms of reducing the number of function evaluations and $a=3.94$ is efficient in attaining an optimal cost almost at every combination of parameters considered in the study. The problem faced with the Logistic sequence with $a=4.0$ is that for the initial values of $0.25,0.5,0.75$, the Logistic equation soon converges to a similar value. And, except for $0.25,0.5$, and 0.75 , for any other initial value, the Logistic sequence converges to zero for a higher iteration size. and SA), in the majority of the cases (i.e., CDGA-I model for BRN and GYN; CDGA-II model for TLN and GYN; and CDGA-III model for TLN), the NSA outperforms the SA in terms of computational efficiency. With the CDGA-II models, for TLN and GYN, the nonsequential $y$ excels in attaining an optimal solution with less computational effort. However, for BRN, the sequential $x$ sequence outperforms the other approaches (see Table 2). While using the CDGAIII model, only the $S$ sequence is efficient in converging to the optimal solution.

The main drawback with the CDGA-III model observed in the present study is the normalization technique used. With the Lorenz map, the difference between the subsequent 
numbers in the series is very small. When the data are normalized to be in the range $(0,1)$, the magnitude of the subsequent values is almost the same for a long stretch. Due to the repetitive values in a stretch, the CDGA-III model with both the approaches does not aid in the diversification or exploration of the search space (selection of an appropriate normalization technique needs to be explored further, and will be done in a future study). Thus, for the convergence to an optimal solution, the CDGA-III model required more computational effort, as is the case for TLN and BRN. The lack of diversification in the search also leads to scenarios where the algorithm is frequently stuck at the sub-optimum solution and is the case with GYN. The same phenomenon is encountered when another higher-dimensional chaotic map, the Mackey-Glass equation is used (results not presented). Also, due to the repetitive values in a stretch, with the CDGA-III model, one more interesting feature observed is that it required more population for the effective search. When large $P_{s}$ are allowed, it is observed that the algorithm converges faster at the early generations, and thus, required less computational time, as reported in earlier sections.

\subsection{Computational Results of GA and CDGA models}

For the three benchmark problems, TLN, BRN, and GYN (whose dimension ranges 8 to 30), the results suggest that introducing the chaos ergodicity in the GA search phenomenon acts as a carrier wave in decreasing the search space, inducing faster convergence to the optimal performance comparison of GA and CDGA models and with the other models reported in the literature is enclosed in the supplementary material, S_4.

Overall, when the CDGA models with different chaotic maps are considered, for the three benchmark problems TLN, BRN, and GYN, the CDGA-I model is found to be the best. 
to be superior over SA. Thus, considering a large 454-dimensional problem, BIN, the

492

493

494

495

496

497

498

499

500

501

502

503

504

505

506

507

508

509

510

511

512

513

514

515

scalability of the best model, the CDGA-I with the NSA is evaluated as follows.

\subsubsection{Scalability of CDGA-I model}

As similar to the other three benchmark problems considered in the study, for BIN also, before the application of the CDGA-I model, sensitivity analysis is performed. The optimal parameter set ascertained from sensitivity analysis is $P_{s}=700, G_{s}=3000, P_{m}=0.01, \mathrm{PM}=$ $1,000,000$, elitism percentage $=20$, and a slightly higher $P_{c}$ of 0.9 . With this combination of parameters, the CDGA-I model with NSA at $a=4.0$, resulted in an improved design cost of $€ 2,145,627$ when compared to $€ 2,302,423$ reported by Reca and Martinez (2006) using the GA model. The MFEs to locate $€ 2,145,627$ is $1,944,600$ which is far lesser than the 10 million that is required for locating $€ 2,302,423$. When the $G_{s}$ is increased to 10,000 , an optimal cost of $€ 2,091,547$ is obtained using the CDGA-I model, requiring 4,565,400 MFEs which is still less than 10 million. When the $P_{s}$ is increased to 2400 by keeping the remaining parameters the same as the ones calibrated, an optimal cost of $€ 2,047,814$ is obtained which differs by $€ 254,609$ from $€ 2,302,423$. The MFEs required to locate $€ 2,047,814$ is $7,024,800$ which is less by $2,975,200$ as compared to 10 million. Also, the optimal cost obtained using the CDGA-I model is far lesser as compared to the design cost of $€ 2.218$ million, reported by Moosavian and Lence (2019) using the fittest individual referenced DE model.

Thus, the computationally best of all the models, the CDGA-I model, successfully proves its scalability on its application to the 454-dimensional problem, resulting in an improved design cost with less computational effort.

\section{Conclusions}

This paper presented the chaos-directed genetic algorithm (CDGA) to replace the random phenomenon of GA, considering two approaches: the non-sequential approach (NSA) and the sequential approach (SA). Three chaotic maps of different complexities and 
dimensions, namely the Logistic map, the Henon map, and the Lorenz map, were considered

517 and their effects on the computational effort of GA was studied by validating them on three

518 benchmark problems: Two-loop network (TLN), Bakryan network (BRN), and Goyang

519 network $(\mathrm{GYN})$. The results revealed that the GA model itself is efficient with reduced

520 computation effort when compared to other widely studied algorithms. The chaos-directed,

521 CDGA-I, and CDGA-II models (with the Logistic and Henon map, respectively) were found

522 to outperform GA with their better search efficacy in reaching the optimum solution with less

523 computational effort. The main advantage of the CDGA-II and CDGA-III models is that if the

524 optimal parameter set is once fixed through a sensitivity analysis, it excels in converging to the

525 optimal solution at every trial. Further, the scalability of the best of all the models is

526 successfully evaluated considering a large dimensional problem, the Balerma irrigation

527 network (BIN).

528 The results from the detailed analysis indicate that the combination of chaos ergodicity

529 with GA heuristics seems to have great potential and can be extended for practical applications.

530 Other bio-inspired or swarm-based metaheuristic algorithms can also be combined with chaos

531 ergodicity to improve their search efficacy with the global perspective to deal with large-scale

532 non-deterministic polynomial-time hard (NP-hard) problems.

\section{Notations}

$534 C_{j}:$ cost of $j^{\text {th }}$ pipe

$535 C D S$ : commercially available diameter set

$536 C P$ : crossover point

$537 C S$ : chaotic sequence

$538 D:$ genes or decision variable

$539 d_{j}$ : diameter of $j^{\text {th }}$ pipe

$540 D L_{s}$ : chaotic sequence data length allocated for a single generation using SA

$541 F_{f}$ : fitness function

$542 G_{s}$ : generation size 
$543 \quad H_{i}$ : head available at the $i^{\text {th }}$ node

$544 H_{\text {min }}$ : minimum head requirement at the $i^{\text {th }}$ node

$545 L$ : lower bound of the decision variable

$546 l_{j}$ : length of $j^{\text {th }}$ pipe

$547 M:$ number of genes that undergo mutation operation

$548 M C:$ mutation chromosome

$549 M V$ : mutation variable

$550 N_{C D}$ : number of commercially available pipe diameter options

$551 \quad N_{D}$ : number of decision variables

$552 \quad N_{n}:$ number of nodes

$553 \quad P C$ : parent chromosome

$554 \quad P_{c}:$ crossover probability

$555 P_{m}:$ mutation probability

$556 \quad P M$ : penalty multiplier

$557 \quad P_{r}:$ number of chromosomes retained during trunctation operator

$558 P_{s}$ : population size

$559 \quad r$ : uniformly distributed random number

$560 S V$ : swapping variable

$561 U$ : upper bound of the decision variable

\section{Declarations}

\section{Funding}

564 Not applicable

\section{Conflict of Interest}

566 The authors declare that they have no conflict of interest.

567 Availability of Data and Material

568 Not applicable

569 Code Availability

$570 \quad$ Not applicable 
Ali MZ, Awad NH, Suganthan PN, Shatnawi AM (2018) An improved class of real-coded genetic algorithms for numerical optimization. Neurocomputing 275(2018): 155-166

Alperovits E, Shamir U (1977) Design of optimal water distribution systems. Water Resources Research 13(6): 885-900

Ammaruekarat P, Meesad P (2011) A chaos search for multi-objective memetic algorithm. 2011 International Conference on Information and Electronics Engineering IPCSIT vol.6, IACSIT Press, Singapore

Arunkumar R, Jothiparakash V (2013) Chaotic evolutionary algorithms for multi reservoir optimization. Water Resources Management 27(15): 5207-5222

Cheng C-T, Wang W-C, Xu D-M, Chau K-W (2008) Optimizing hydropower reservoir operation using hybrid genetic algorithm and chaos. Water Resources Management 22(7): 895909

Cimorelli L, D'Aniello A, Cozzolino L (2020) Boosting genetic algorithm performance in pump scheduling problems with a novel decision-variable representation. Journal of Water Resources Planning and Management 146(5): 04020023-1 - 04020023-11

Cisty $M$ (2010) Hybrid genetic algorithm and linear programming method for least-cost design of water distribution systems. Water Resources Management 24(1): 1-24

590 Dandy GC, Simpson AR, Murphy LJ (1996) An improved genetic algorithm for pipe network optimization. Water Resources Research 32(2): 449-458

Ezzeldin R, Djebedjian B, Saafan T (2014) Integer discrete particle swarm optimization of water distribution networks. Journal of Pipeline Systems Engineering and Practice 5(1): 04013013

Fallah H, Kisi O, Kim S, Rezaie-Balf M (2019) A new optimization approach for the least-cost design of water distribution networks: An improved crow search algorithm. Water Resources Management 33: 3595-3613

Geem ZW (2006) Optimal cost design of water distribution networks using harmony search. Engineering Optimization 38 (3): 259-277

Gessler J (1985) Pipe network optimization by enumeration. In: Proceedings of Computer Applications in Water Resources, ASCE, New York, N.Y. 572-581

602 Goldberg DE, Kuo CH (1989) Genetic algorithms in pipeline optimization. Journal of 603 Computing in Civil Engineering 1(2): 128-141

604 Grassberger P, Procaccia I (1983) Measuring the strangeness of strange attractors. Physica D, 605 9(1-2), 189-208

606 Gupta I, Gupta A, Khanna P (1999) Genetic algorithm for optimization of water distribution 607 systems. Environmental Modelling and Software 14(5): 437-446 
Henon M (1976) A two-dimensional mapping with a strange attractor. Communications in Mathematical Physics 50(1): 69-77

612 Ingu T, Takagi H (1999) Accelerating a GA convergence by fitting a single-peak function. 6131999 IEEE International Fuzzy Systems Conference Proceedings, Seoul, Korea, III- 1415: III6141420

615 Johns MB, Keedwell E, Savic D (2014) Adaptive locally constrained genetic algorithm for 616 least-cost water distribution network design. Journal of Hydroinformatics 16(2): 288-301

617 Kadu MS, Gupta R, Bhave PR (2008) Optimal design of water networks using a modified 618 genetic algorithm with reduction in search space. Journal of Water Resources Planning and 619 Management 134(2): 147-160

Keedwell E, Khu ST (2005) A hybrid genetic algorithm for the design of water distribution networks. Engineering Applications of Artificial Intelligence 18(4): 461-472

Kim JH, Kim TG, Kim JH, Yoon YN (1994) A study on the pipe network system design using non-linear programming. Journal of Korean Water Resources Association 27(4): 59-67

Lansey KE, Mays LW (1989) Optimization model for water distribution system design. Journal of Hydraulic Engineering 115(10): 1401-1418

Lee SC, Lee SI (2001) Genetic algorithms for optimal augmentation of water distribution networks. Journal of Korean Water Resources Association 34(5): 567-575

Lorenz EN (1963) Deterministic nonperiodic flow. Journal of the Atmospheric Sciences 20: 130-141

May RM, (1976) Simple mathematical models with very complicated dynamics. Nature 261: 459-467

Michalewicz Z, Schoenauer M (1996) Evolutionary algorithms for constrained parameter optimization problems. Evolutionary Computation 4(1): 1-32

Montesinos P, Garcia-Guzman A, Ayuso JL (1999) Water distribution network optimization using a modified genetic algorithm. Water Resources Research 35(11): 3467-3473

Moosavian N, and Lence B (2019) Fittest individual referenced differential evolution algorithms for optimization of water distribution networks. Journal of Computing in Civil Engineering, 33(6), 04019036

Mora-Melia D, Iglesias-Rey PL, Martinez-Solano, Fuertes-Miquel VS (2013) Design of water distribution networks using a pseudo-genetic algorithm and sensitivity of genetic operators. Water Resources Management 27: 4149-4162

Mozaffari A, Emami M, Fathi A (2018) A comprehensive investigation into the 643 performance, robustness, scalability and convergence of chaos-enhanced evolutionary 644 algorithms with boundary constraints. Artificial Intelligence Review 52(4): 2319-2380 No. R93, University of Adelaide, Australia. network - using genetic algorithm and differential evolution. Water Supply 20(1): 95-102 
649 Reca J, Martinez J (2006) Genetic algorithms for the design of looped irrigation water 650 distribution networks. Water Resources Research 42(5): W05416

651 Rossman LA (2000) EPANET 2 USERS MANUAL, U.S. Environmental Protection Agency, 652 Washington, D. C., EPA/600/R-00/057

653 Savic DA, and Walters GA (1997) Genetic algorithms for least-cost design of water 654 distribution networks. Journal of Water Resources Planning and Management 123(2): 67-77

655 Simpson AR, Dandy GC, Murphy LJ (1994) Genetic algorithms compared to other techniques 656 for pipe optimization. Journal of Water Resources Planning and Management 120(4): 423-443

657 Sivakumar B (2017) Chaos in Hydrology: Bridging Determinism and Stochasticity. Springer

658 Vairavamoorthy K, Ali M (2000) Optimal design of water distribution systems using genetic 659 algorithms. Computer-Aided Civil and Infrastructure Engineering 15(5): 374-382

660 Vairavamoorthy K, Ali M (2005) Pipe Index Vector: A method to improve genetic-algorithm661 based pipe optimization. Journal of Hydraulic Engineering 131(12): 1117-1125

$662 \mathrm{Wu}$ ZY, Simpson AR (2001) Competent genetic-evolutionary optimization of water 663 distribution systems. Journal of Computing in Civil Engineering 15(2): 89-101

664 Yuan X, Yuan Y, Zhang Y (2002) A hybrid chaotic genetic algorithm for short-term hydro 665 system scheduling. Mathematics and Computers in Simulation 59: 319-327

666 Zheng F, Simpson AR, Zecchin AC (2011) A combined NLP-differential evolution algorithm 667 approach for the optimization of looped water distribution systems. Water Resources Research 668 47: 1-18

669 Zheng F, Simpson AR, Zecchin AC (2014) Coupled binary linear programming-differential 670 evolution algorithm approach for water distribution system optimization. Journal of Water 671 Resources Planning and Management 140(5): 585-597

672 Zyl JEV, Savic DA, Walters GA (2004) Operational optimization of water distribution systems 673 using a hybrid genetic algorithm. Journal of Water Resources Planning and Management 674 130(2): 160-170 
Table 1. Overall review of benchmark problems considered in the study and the results with the GA, CDGA-I (with the Logistic map), and CDGA-II (with the Henon map) models along with their design parameters

\begin{tabular}{|c|c|c|c|c|c|c|c|c|c|}
\hline \multirow{2}{*}{ Parameter } & \multicolumn{3}{|c|}{ TLN } & \multicolumn{3}{|c|}{ BRN } & \multicolumn{3}{|c|}{ GYN } \\
\hline & GA & CDGA-I & CDGA-II & GA & CDGA-I & CDGA-II & GA & CDGA-I & CDGA-II \\
\hline Number of pipes & \multicolumn{3}{|c|}{8} & \multicolumn{3}{|c|}{9} & \multicolumn{3}{|c|}{30} \\
\hline Commercially available options & \multicolumn{3}{|c|}{14} & \multicolumn{3}{|c|}{11} & \multicolumn{3}{|c|}{8} \\
\hline Solution space & \multicolumn{3}{|c|}{$14^{8}\left(1.475 \times 10^{9}\right)$} & \multicolumn{3}{|c|}{$11^{9}\left(2.357 \times 10^{9}\right)$} & \multicolumn{3}{|c|}{$8^{30}\left(1.237 \times 10^{27}\right)$} \\
\hline Minimum residual pressure $(\mathrm{m})$ & \multicolumn{3}{|c|}{30} & \multicolumn{3}{|c|}{15} & \multicolumn{3}{|c|}{15} \\
\hline Population size $\left(P_{s}\right)$ & 102 & 102 & 53 & 102 & 53 & 53 & 53 & 53 & 53 \\
\hline Probability of crossover $\left(P_{c}\right)$ & 0.5 & 0.5 & 0.7 & 0.5 & 0.7 & 0.7 & 0.5 & 0.7 & 0.7 \\
\hline Probability of mutation $\left(P_{m}\right)$ & 0.02 & 0.02 & 0.03 & 0.035 & 0.06 & 0.06 & 0.025 & 0.05 & 0.035 \\
\hline Generation size $\left(G_{s}\right)$ & 400 & 130 & 400 & 500 & 100 & 100 & 500 & 500 & 500 \\
\hline Penalty multiplier $(P M)$ & \multicolumn{3}{|c|}{$7.5 \times 10^{4}$} & \multicolumn{3}{|c|}{$5 \times 10^{5}$} & \multicolumn{3}{|c|}{$3 \times 10^{7}$} \\
\hline Optimal feasible cost & \multicolumn{3}{|c|}{ US\$ 419,000 } & \multicolumn{3}{|c|}{ US\$ 903,620 } & \multicolumn{3}{|c|}{$177,009,557 \mathrm{KRW}$} \\
\hline Minimum functional evaluations & 3000 & 1600 & 1664 & 2200 & 728 & 1040 & 10088 & 7280 & 22256 \\
\hline Solution space explored (\%) & $\begin{array}{l}2.033 \\
\times 10^{-4}\end{array}$ & ${ }_{4}^{1.084} \times 10^{-}$ & $\begin{array}{c}1.128 \times \\
10^{-4}\end{array}$ & $\begin{array}{l}9.330 \\
\times 10^{-5}\end{array}$ & $\begin{array}{l}3.087 \\
\times 10^{-5}\end{array}$ & $\begin{array}{l}4.411 \\
\times 10^{-5}\end{array}$ & $\begin{array}{c}8.149 \times \\
10^{-22}\end{array}$ & $\begin{array}{l}5.881 \\
\times 10^{-22}\end{array}$ & $\begin{array}{c}1.798 \times \\
10^{-21}\end{array}$ \\
\hline Average computation time (s) & 21.556 & 6.917 & 8.007 & 74.988 & 8.003 & 8.035 & 27.352 & 27.002 & 25.985 \\
\hline
\end{tabular}




\begin{tabular}{|c|c|c|c|c|}
\hline $\begin{array}{c}\text { Benchmark } \\
\text { problem }\end{array}$ & $\begin{array}{c}\text { Different approaches to } \\
\text { CDGAII }\end{array}$ & $\begin{array}{c}\text { Minimum } \\
\text { functional } \\
\text { evaluations }\end{array}$ & $\begin{array}{c}\text { Time of computation } \\
(\mathrm{s})\end{array}$ & Optimal parameter set \\
\hline \multirow[t]{4}{*}{ TLN } & Non-sequential $x$ & 1820 & 8.972 & $P_{s}=53, G_{s}=400, P_{m}=0.04, P_{c}=0.7$ \\
\hline & Non-sequential $y$ & 1664 & 8.007 & $P_{s}=53, \mathrm{G}_{\mathrm{s}}=400, P_{m}=0.03, P_{c}=0.7$ \\
\hline & Sequential $x$ & 3016 & 11.228 & $P_{s}=53, G_{s}=400, P_{m}=0.05, P_{c}=0.7$ \\
\hline & Sequential $y$ & 1924 & 9.852 & $P_{s}=53, G_{s}=400, P_{m}=0.02, P_{c}=0.5$ \\
\hline \multirow[t]{4}{*}{ BRN } & Non-sequential $x$ & 1352 & $8.071,8.440$ & $P_{s}=53, G_{s}=100, P_{m}=\{0.06,0.07\}, P_{c}=0.7$ \\
\hline & Non-sequential $y$ & 1248 & 8.704 & $P_{s}=53, G_{s}=100, P_{m}=0.1, P_{c}=0.7$ \\
\hline & Sequential $x$ & 1040 & 8.035 & $P_{s}=53, G_{s}=100, P_{m}=0.06, P_{c}=0.7$ \\
\hline & Sequential $y$ & 1196 & 8.075 & $P_{s}=53, G_{\mathrm{s}}=100, P_{m}=0.1, P_{c}=0.7$ \\
\hline \multirow[t]{4}{*}{ GYN } & Non-sequential $x$ & 28000 & 67.775 & $P_{s}=204, G_{s}=300, P_{m}=0.04, P_{c}=0.7$ \\
\hline & Non-sequential $y$ & 22256 & 25.985 & $P_{s}=53, G_{s}=500, P_{m}=0.035, P_{c}=0.7$ \\
\hline & Sequential $x$ & 26400 & 67.278 & $P_{s}=204, G_{s}=300, P_{m}=0.035, P_{c}=0.7$ \\
\hline & Sequential $y$ & 29000 & 69.326 & $P_{s}=204, G_{s}=00, P_{m}=0.03, P_{c}=0.7$ \\
\hline
\end{tabular}




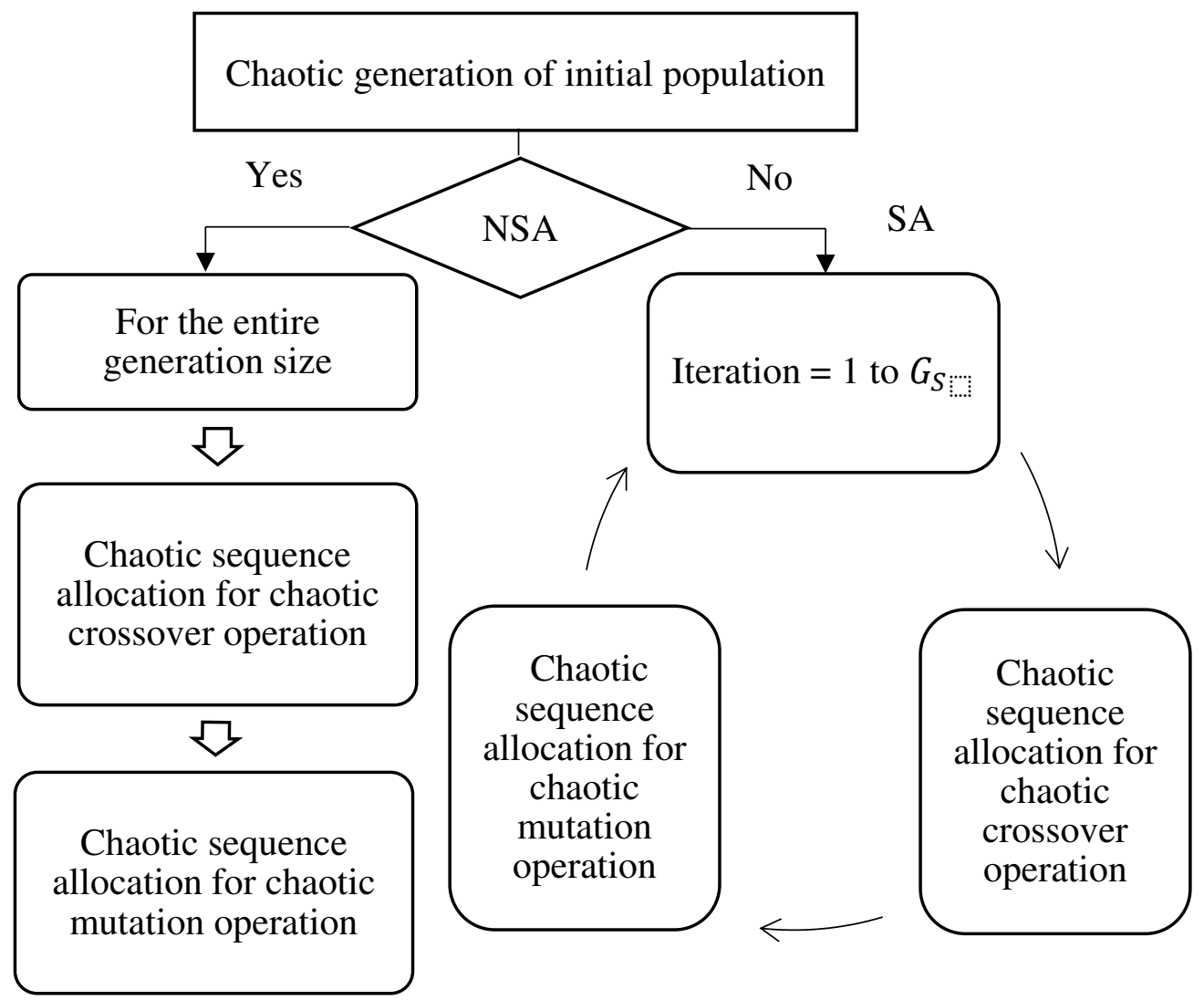

Fig. 1. Mechanism of the non-sequential and sequential approaches considered in this study 
CDGA-I
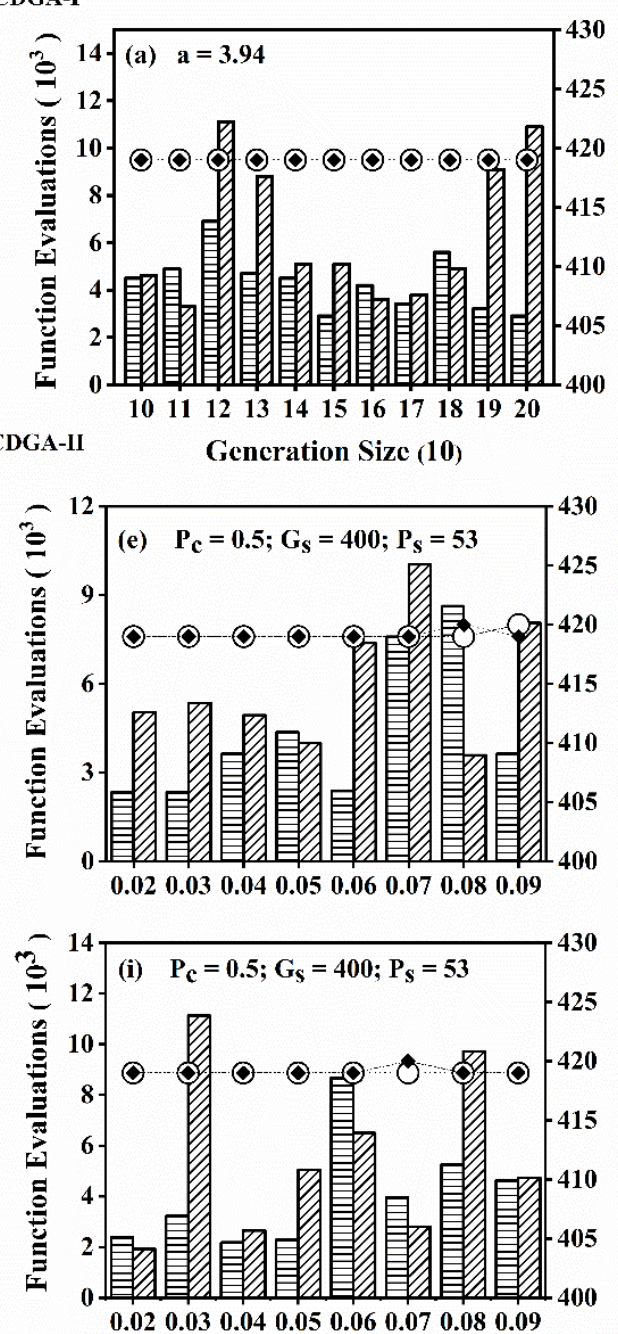

Probability of Mutation

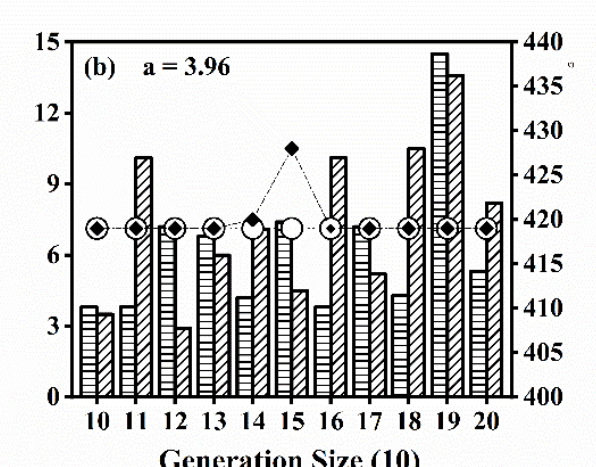

Generation Size (10)
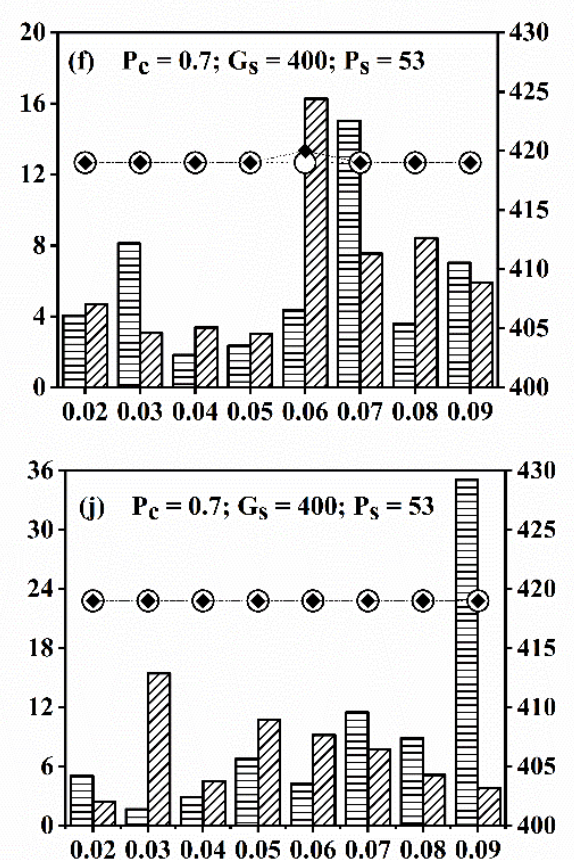

Probability of Mutation

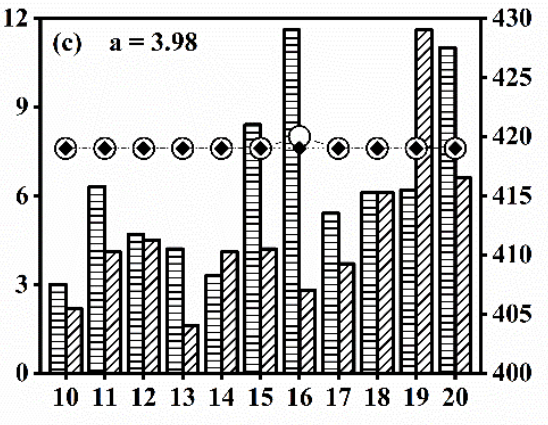

Generation Size (10)
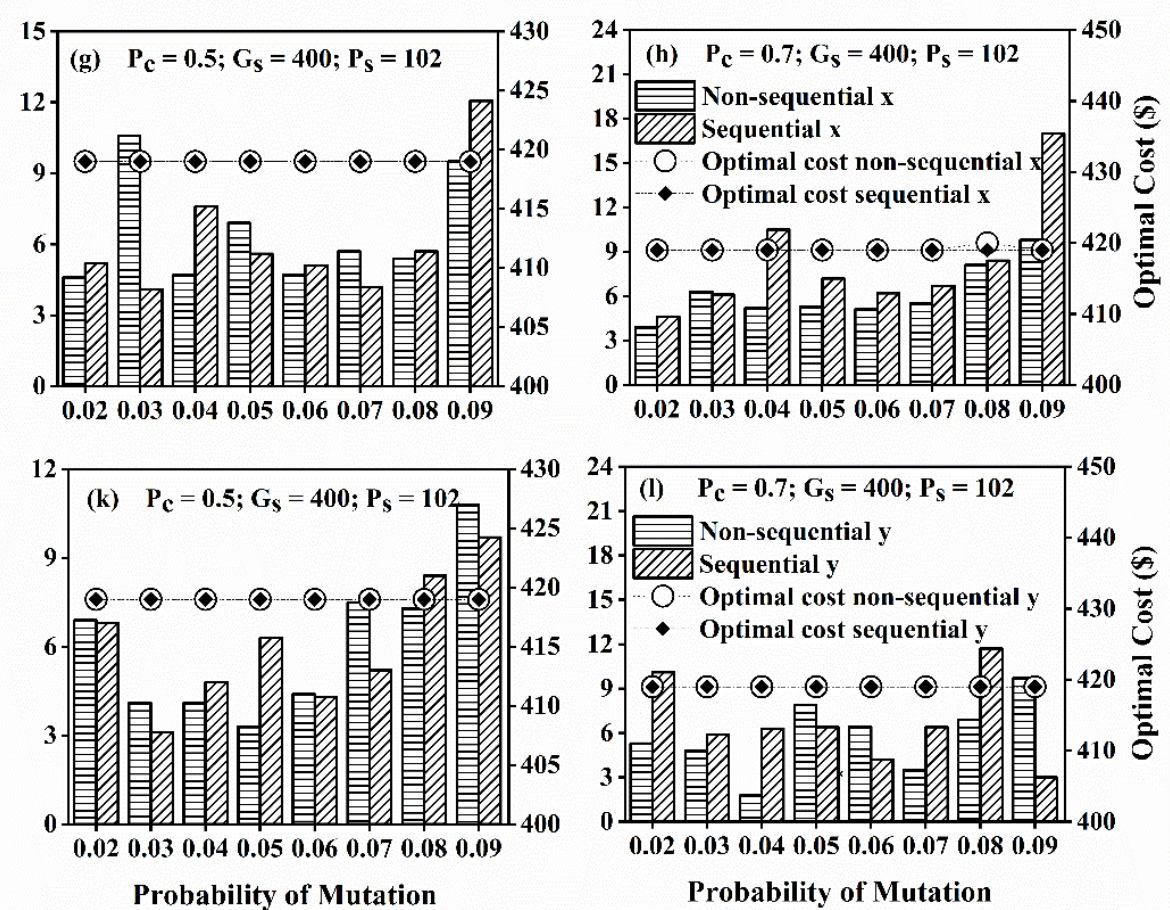

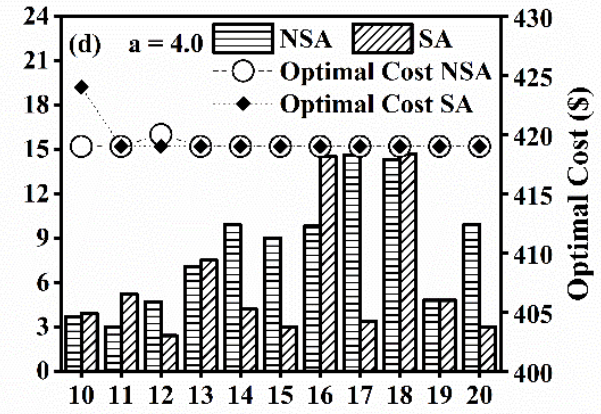

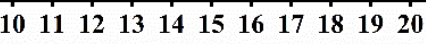

Fig. 2 Computational results for TLN using CDGA-I and CDGA-II models 


\section{Supplementary Files}

This is a list of supplementary files associated with this preprint. Click to download.

- Supplementarymaterial.docx 